\title{
La discusión sobre las artes y oficios en los albores de la república
}

\begin{abstract}
Durante los inicios de la vida independiente, más especificamente en el tramo comprendido entre 1810 y comienzos de la década de 1830 , dentro del gran debate quie sentó las bases para la institucionalidad de la república se asignó interés al desarrollo de las artes y oficios en el contexto chileno, lo que era visto por un lado como camino a la "regeneración del pueblo" a través de la educación popular; y por otro, como camino al desarrollo productivo mediante la formación de recursos humanos que pudicran encontrar ocupación como artesanos u obreros. Este artículo aborda el escenario previo a la instauración de esta enseñanza en el país, lo que tuvo lugar a fines de la década de 1840 con la apertura de la Escuela de Artes y Oficios de Santiago.
\end{abstract}

Palabras clave: educación tecnológica, artes y oficios. Chile

Desde los últimos tiempos de la Colonia hasta los albores de la vida independiente, distintos autores clave en el proceso de emancipación como Juan Egaña, Fray Camilo Henríquez y Manuel de Salas abordaron en forma crítica el atraso cultural en la Capitanía General de Chile. Este último, uno de los precursores del pensamiento ilustrado en el país, señalaba en uno de sus escritos políticos de la época (1810): "nos han mantenido en la oscuridad y en la miseria..." 'La sentencia del destacado intelectual y patriota aludía tanto a la pobreza intelectual como material del medio chileno, donde además del escaso progreso de las letras, era reconocible también un pobre conocimiento acerca de los adelantos técnicos que en Europa y Estados Unidos se estaban desarrollando a causa de la Revolución Industrial ${ }^{2}$. Al 
respecto, el mismo auzor se había referido de esta forma al desarrollo alcanzado por los oficios en el país a fines de la Colonia (1796):

Herreros coscos, plareros sin gusto, carpinteros sir principios, albaniles sin arquirec. tari. pineores sin dibujo, sastres imitadores. bencticiadores sin docimasia, - hoja lateros de rutina zapatcros cramposos, torman la caterva de arresanos que cuanto hacen a cientas mis lo deben á la afición y a la necesidad de sufrirtos que a un arreglado aprendizajc... Su ignorancia, las pocas urilidades y los vicios que son consiguicntes les hacen desertar con frectencia, y. variando de profesiones, no tener ninguma. Si por medio de una academia ó sociedad se les inspirasen conocimiencos y una noble comulación. cllos se estimaran distinguinian desde lejos el termino á que preden liegar y emprerdiendo el camino, scrian constantes, driles y acomodados: tal vez harim brotar de cada are los ramosen que esrin divididas en los hugares donde se ham perteceionado."

Esta conjunción de prènisas (el afán educacional; el afán científicotecnológico) motivó que a poco andar del gobierno liderado por José Miguel Carrera, el debate sobre la necesidad de fomentar tanto el desarrollo industrial como la instrucción de orden práctico cobraza protagonismo entre las esferas dirigentes del país. Un artículo publicado en la Aurora de Chile por Manuel Gandarillas (1812), se refería al panorama educacional chileno en los siguientes términos:

\footnotetext{
Y que cotanzos atrasados es tanta verdad... La arzy yuntamenco de todas las ciencias es cel lece excribir y contar artes necesarias para civilizar a los pucblos y dirigirlos á su grandeza y con todo ignondias, o poce sabidas de lo gencral de la nacion. No sola. mente los nobles y los ricos deberian ser doctrinados en estos principios, sino los plebeyos. los aresanos, los kabradores... ${ }^{4}$
}

En el mismo texto el autor indicaba entre las causas de la situación "que no se cultivan bien los ingenios, ni se ilustra la razón" al tiempo "que las artes primitivas y secundarias, se hallan imperfectas". Como alternariva, Gandarillas proponía la acción que el gobierno debia realizar al hacerse cargo del desarrollo general de la educación, hasta entonces principalmente en manos de congregaciones religiosas. La concinuación del mismo artículo en la edición siguiente del periódi$c o$, señalaba que la acción gubernamental debía encaminarse "en las funciones de los Colegios, y Seminarios, en donde se eduquen los jóvenes, y en los establecimientos de Casas públicas, en donde los hijos de la plebe aprenden las artes mecánicas, corrigen sus costumbres y cultivan sus modales". "De acuerdo a este último pasaje, en el primer caso se vislumbraba a la educación privada y a la universidad como el recinto a ocupar por los hijos de la elite local, mientras que los hijos del pueblo encontrarían en planteles de orden público tanto una 
instrucción práctica como la formación ciudadana. Otro artículo, publicado en el primer periódico nacional a mediados del mismo año, continuaba la discusión esbozando además una crítica a las elites cconómicas por su desinterés ante el escaso desarrollo educacional y productivo que a la fecha alcanzaba el contexto chileno:

El pucblo vive en pobrcza, cin miscria en medio de la mayor abundancia: las primeras materias de las artes o se pierden, o no producen codas las ventajas posibles: la ociosidad de la plebe cs lascimosa: la agricultura por si sola no emplea á todos los hombres ni en rodos los tiempos: las mugeres, Los ninos. los viejos no tienen disposiciones para sus fatigas: la muger, las hijas del labrador le son una carga pesada, por que no hay fabricas en que ocuparse: los propictarios son pocos: jcomo podran los jornaleros mantener a sus fanilias, si casi esean en la clase de mendigo? Esre es al dolorido clamor de nuestros policicos. Ellos todo lo dificulan, sin dar un paso para venect las dificultades. Quisioran que en el dia apareciesen lienzos tinos y ricas cstofas trabajadas en cl pais, sin advertir, que esco no esta en el orden de la naruralezat que es preciso, que las telas bastas precedan á las fimas y las obras ordinarias a las de un gusto exquisito. Quisictan. que cl pucblo sin arbierios. sin candal. y sin luces emprehendicse los cstablecimientos costosos de las artes. Estas empresas son propias de los hombres ricos: pero es cosa lamentable, que los que pueden enscriar los trabajos urikes, y enriqueser al pais, no hallen en los ricos d fomento y la procecion, de que necesiran. ${ }^{*}$

El artículo denotaba gran parte de la discusión que recorrió el siglo XIX y gran parte del $\mathrm{xx}$, donde términos como instrucción práctica, manufacturas, fomento, oficios, educación popular fueron actores que se intentó alinear en mayor o menor medida, y con distinta suerte en diferentes etapas de la historia nacional; pero en los inicios de la vida independiente la circulación de cstas ideas pudo expresarse a través de la Sociedad Económica de Amigos del País, organismo cuya fundación en 1813 reclamaba "que busque todos los medios de promover la industria, y haga familiares los más importantes descubrimientos" ${ }^{8}$. Otro de los argumentos para la creación de esta entidad era que "todas las naciones cultas han conocido, que la agricultura, las artes necesitan sociedades politicas, que las fomenten, y cuiden de su enseñanza, y perfeccion" 9 . Justamente, la conformación de este tipo de organizaciones en el mundo respondía al afán proveniente de la llustración en cuanto a promover el desarrollo y la difusión de la cultura hacia todos los ciudadanos, y uno de los autores gravitantes a nivel nacional en el círculo dirigente animado por estas ideas fue Pedro Rodríguez de Campomanes ( $7723-1803$ ), político y economista español que fuera nombrado ministro de Hacienda por el gobierno de Carlos III en 1760 , y que 
en su Discurso sobre el fomento de la industria popular (1774) propuso el énfasis en la enseñanza industrial y la creación de manufacturas locales como camino para el progreso, al tiempo que planteaba la necesidad de dar al gobierno y a las escuelas públicas el rol de difundir las artes y oficios, relevando de esta forma el protagonismo que hasta entonces tenían los gremios, por quedar reducido a los interereses o el radio de acción de estos pequeños grupos aspectos que implicaban una preocupación o responsabilidad a nivel de los países:

Nada es mas contrario i la industria populax, que la creccion de gremios y fucros privilegiados: dividiendo en unas sociedades pequenas al problo y eximiendolas te la justicia ordinaria en mechos casos. Si este metodo se repiec denasiado son de eener consecuencias desagradables contra la extension y bondad de las manuficteuras. ${ }^{\text {to }}$

El fomenco de las ares es incomparible con la subsistencia imperfeca de gremios: clles hacen cstanco de los oficios, yá titulo de ser únicos y privarivos, no se toman la fatiga de esmerarse en las ares; por que saben bien, que el publico los ha de buscar necesariamence y no se pira en discernir sus obras. ${ }^{\text {t }}$

Otra de las premisas de este autor fue el énfasis dado a la relación entre arte y ciencia como camino para el desarrollo productivo, lo primero, por atender aspectos como el gusto o la sensibilidad tanto de los artesanos como del público; lo segundo, por ser el camino para asimilar las nuevas técnicas o inventos, donde respectivamente señalaba la importancia que se debía asignar tanto al dibujo como a las matemáticas en el establecimiento de las artes y oficios:

En los gremios de aresanos hay poquísima crseñanza. Fada dibujo en los aprendices. escucha publica de cada oficio, y premios á los que akclanten, ó nejoren la profesióm." ${ }^{2}$

Las maremáticas son las que taciliean cl conocimienro, la invención y la perfeccion de las náquitras para cmplcarlas cn todas las arces y oficios. Por la misma razon deberia dorarse a lo menos un Maestro ó Caredrático de matemática con un buen salario cn la misma Capical de la Provincia y alli deberia dar leccion a quantos las quisiesen aprender. y resolver las dudas que ocumiesen aplicativas a las arecs, y a sus inscrumencos, máquinas, y usos sujcros al cálculo. ${ }^{1 ;}$

Otro de los textos referenciales de Campomanes fue su Discurso sobre educación popular de los artesanos y su fomento $(x 775)$ y entre sus ideas centrales destacaba la importancia asignada al dibujo, que el autor consideraba como "el padre de los oficios prácticos, y sin él nunca podran florecer" ${ }^{14}$. Para esto, sugería como alternativa instalar escuelas de dibujo bajo la tutela de las sociedades de amigos del país, consignando además la importancia de que este aprendizaje fuese complementario al trabajo de los artesanos: 
Lar horas de csta csanka... Leberian scr acomodadas, y discrineas de las en que trabajan los artifices parano inofedir álos aprendices la asistencia a los talleres de sus maestros.. Estos no solo no han de poder impedir.que sus aprendices veyan ála cscucla de dibujo: sino zchar cl que necesariamence concuran sin escusa, ni falta algurna. ${ }^{\text {.5 }}$

Campomanes entendia al dibujo como un lenguaje que podía favorecer el desarrollo de la industria más allá de la relación entre maestro y aprendiz proveniente de la tradición de los gremios:

Las artes y oficios. que innediatanente no necesicen cl dibuxo, se ven precisadas a valerse de d. para dar a conocer sus instruncntos, miquimas, yoperaciones: por chyo medio se hacen perceptibles rá los que no las salyen ni profesan. ${ }^{16}$

Al referirse al término "artesy oficios", el autor buscaba realizar un distingo entre lo que implicaba uno u otro concepto, que unidos se tradujeron en una denominación nueva, afín al espíritu de la Revolución Industrial. Campomanes señalaba a las artes como "las que necesitan de reglas y aprendizage" ${ }^{17}$ mientras que los oficios "no necesitan de reglas, y les basta la pura imitacion, disposicion natural y fuerzas". "De esta forma, entendía "que en el modo comun de hablar, se suele denominar á las artes oficios, porque en realidad todo arte es oficio; pero no al contrario" ". Y en la relación entre uno y otro, el dibujo tenía un rol central.

A nivel local, en los inicios de la vida independiente se vislumbraba que el desarrollo industrial traería consigo el desarrollo económico y en consecuencia el bienestar de la sociedad chilena, pero que dicho proceso requería la presencia de maestros de los que a la fecha carecía el país:

La industria trahe las riquezas y las riquezas forman cl poder nacional. La indestria incroduce el trabajo; y el rabajo desticrnalocio yálos vicios. Los pueblos laboriosos eienen costumbers. La riqueza. y las coscumberes son dapoyo, el recurso d baluare de ha libertad, Como pues tan de ontitirsc los medios indispensables. para llamar la industria a nuestro territorio? COno no han de dictarse todas las precisas providen. cias. y removerse todos los obstaculos, para atraher. y domiciliar entre nosoeros los macstros de las attes? 20

Sin embargo, en la discusión de la época accrca del establecimiento de la enseñanza industrial en el país estaba clara como una de las dificultades a enfrentar el desdén por el trabajo manual y los oficios, lo que se atribuía a la falta de cultura:

El deshonor afecto a las profesiones mecanicas retrahe del trabajo c introduce, $y$

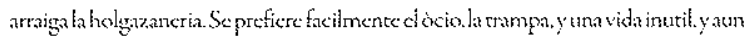
perniciosá un trabajo que es honesto en los pucbios cultos pero que envilece en los obscurecitos y csupjidos. Un pubblo sugcto a la funesta intluencia de semejante errores jamas saldra de ha miscria.y el abatinvicuro: añadamos aun, janzas serà libre. "r 
Frente a este desprestigio social, la conformación de la Sociedad Económica de Amigos del País, y el futuro establecimiento por su intermedio de la enseñanza de los oficios a nivel nacional, eran vistos como relevo de los gremios artesanales provenientes de la Colonia, y asi también de su estratificación del trabajo, para lo cual se invocaba en el artículo publicado en la Aurora de Chile una cita al Discurso sobre el fomento de la industria popular:

La introduccion de artifices excrangeros es uno de los fomentos mas seguros de la industriat con ellos se pueden tener materos idoneosen las Provincias, paria propagar la cnscnanza; sugecando a clla a los individuos actuales de los mismos (iremios, que necesiten de este auxilio, por falarles á mechos dibujo el aprendizagenecesario, y un riguroso examen publico, que acredite su suficiencia.

El adekantamicnto de las arecs y oficios ha de ser quieando esrancos; y dando premios a los que sobresalgar a costa de los caudales públicos ó de los Gremios de artesanos cque tengan rentas y fincis.

Es también necesurio borrar de los oficios rodo deshonor: y habiliará los que los exercen para los empleos municipales de la Republica... Solo la holgazaneria debe coneraher la vileza. ${ }^{22}$

En 1813, un nuevo artículo publicado en la Aurora de Chile se refería al plan de organización de la Sociedad Económica de Amigos del País y su reciente exposición ante las autoridades de la época. Entre las premisas del nuevo organismo estaba trabajar por el fomento de actividades productivas como la agricultura y la ganadería, y así también promover el cultivo de

la industria popular i los oficios... los secrecos de las arees, has maquinas, ila cducacion de todas las clases del Escedo, en rok bo lo que renga relacion con la economia politica induscrial ${ }^{23}$

Para ello, entendía la sociedad que entre sus atribuciones estaba normar "las ordenanzas jenerales i particulares de los gremios" :-, contando con la autoridad para ozorgar "los títulos de maestros i oficiales en todas las artes i oficios, como a aquel cuerpo que tiene a la vista el mérito de todos; i estos documentos se darán sellados con el sello de la Sociedad" "s. Como incentivo al mejoramiento de la calidad en la práctica de los oficios, se contemplaba "dar cada año ciertos premios a los artesanos, los que se distribuirán entre los que mejor desempeñasen una obra encomendada" ${ }^{\prime}$. Para el cumplimiento de la tarea propuesta, se consideraba también de importancia la difusión del conocimiento sobre las artes y oficios por medio de impresos, al tiempo que se hablaba también entre los estatutos de la sociedad del establecimiento de "escuelas patrióticas" 
destinadas a la educación de la juventud, concepto que provenía también del Discurso sobre la educación popular de los artesanos y su fomento, escrito por Campomanes a fines del siglo xvir.

Otro artículo publicado en la Aurora de Chile señalaba que con fecha 25 de enero de 1813 se celebró la apertura de la Sociedad Económica de Amigos del País ${ }^{77}$, ocasión en donde su secretario José Antonio de Irisarri expresó los siguientes conceptos en el discurso inaugural de la organización:

\footnotetext{
La tierm abrirà su seno avaro para sutisfacer las necesidades de todos los habitantes de Chile sin distincion de clases ni forumas. Elarte proporcionarà los medios de adequirir todas las comodidades de la vida. La ilustracion disipara las sombras de da ignorancia, y los dias mas claros mas de liciosos y serenos. seguiran a las noches tenebrosas en quec estubicron envuctas nucstras vidas. ${ }^{28}$
}

Pese al promisorio futuro que vislumbraba la entidad, el anhelo ilustrado de fornentar el progreso del país por medio de la enscñanza y práctica de "las artes mecánicas y útiles" tuvo que esperar varias décadas, primero a causa de los agitados años de la emancipación chilena, proceso extendido entre 18.4 y 1823 , y posteriormente a causa de la búsqueda de una institucionalidad política para la nueva república, período que culminó en 1830 con la instauración del sistema autoritario que condujo al país por cuatro décadas ${ }^{29}$. No en vano, en 1821 los ideales provenientes de la sociedad económica fundada en los inicios de la vida republicana reaparecieron en una nueva organización, la Sociedad de Amigos del País, que en su acta de constitución ${ }^{30}$ expresaba la voluntad de

inspinar gradualmente el anor a las ciencias, proteger las artes, velar sobre la educacion de los jóvenes, agenciar los medios de eseablecer itsciruciones piadosas y tocar gencralnente todos los resorres de ha felicidad publica. ${ }^{3 *}$

La entidad, a diferencia del sentido que había animado la discusión una década atrás, dejaba ver en su discurso que el escaso desarrollo productivo del país podía traducirse en inestabilidad social y política, donde

se cstár viendo con dolor los campos incultos, Las minas abandonadas los hombres ociosos y consiguicntementc, ellos y sus familias abutritos por la inacción y ahrumados por la miseria: de cuyos principios se siguen casi de necesidad ha relajacion de las costumbers, cl olvido de eoda moral y cl rompimiento de rodos los lazos que constiruyen a los hombres en sociedad, felices y contentos."

Respecto a la situación descrita, la sociedad consideraba que esta provenía 
en gran parte de la ignorancia y de la falta de estinulo que los cindadanos de rodas clascs y condiciones necesizan para csforzar el ingenio y aplicar los brazos a las tareas gue pucdanpropocionarks supropio bicn y contribur al de los demas.. ${ }^{33}$

Sin embargo, fue recién a mediados del siglo XIX y a fines del pexíodo presidencial de Manuel Bulnes, que la discusión proveniente desde los albores de la vida nacional respecto a la necesidad de instauxar la enseñanza industrial en el país tuvo su expresión en un proyecto educativo de carácter gubernamental, que combinó el afán del desarrollo industrial, al de la "regeneración del pueblo" proveniente de la visión ilustrada. ${ }^{34}$

\section{Notas}

1. "Diálogo de los porteros". En: Universidad de Chile. 1914. Escritos de don Manuel de Salas y documentos relativos a \&l y su familia, tomo Il. Santiago, Chile: Imorenta Barcelona, p. 138. La atención a este pasaje proviene de Bemardo Subercaseaux en su referencial trabajo Historia del libro en Chile.

2. En cuyo desarrollo tuvo protagonismo desde mediados dei sigio XXX ta vision positivista, en cuanto esta corriente filosótica reconocía al conocimiento cientílico como el saber válido u objetivo, al tiempo que 6 método cientitico y las matemálicas cono ciencia exacta tuvieron su correlato en las máquinas a vapor primero y eléctricas después, que hicieron posible el paso de la producción artesanal a la industrial.

3. "Representación hecha al ministro de Hacienda don Diego de Gardoqui por el Síndico del Real Consulado de Santiago, sobre el estado de la agricultura, industria y comercio del Reino de Chile". En: Universidad de Chile, op cit., p. 171.

4. Gandarilas, Manuel. 1812. "Educacion". Aurora de Chile, tomo I núm. 9, Santiago, Chile, jueves 9 de abril, p. 37 .

5. Hbid.

6. Gandarillas, Manuel. 1812. "Continua el Discurso sobre la Educacion". Aurora de Chile, tomo l múm. 10, Santiago, Chile, jueves 16 de abril, p. 44

7. Sin título. 1812. Aurora de Chile, tomo inúm, 23, Santiago, Chile, jueves 16 de julio, p. 93. Este articulo, que ha sido atsibuido a Camilo Henriquez, no lleva autoria.

8. Ibíd.

9. Ibíd. p. 94 .

10. Campomanes, Pedro Rodríguez de. 1774. Discursa sobre el fomento de la industria popular. Madrid, España: Imprenta de Antonio de Sancha, p. cix.

11. lbid., pp. cxvi-cxili

12. Hid., p. cix.

13. Hoid., p. xxxviii.

14. Campomanes, Pedro Rodriguez de. 1775. Discurso sobre la educacion popular de los artesanos y su fomento. Madrid, Imprenta de Anionio de Sancha, p. 56.

15. Jbid., p. 113 .

16. Jbid., p. 111.

17. bíd., p. 99.

18. bid., p. 98. 
19. Ibid. p. 99.

20. Sin titulo. 1812. Aurora de Chile, tomo I núm. 23, Sanilago, Chile, jueves 16 de julio, p. 94.

21. libid.

22. Campomanes, Pedro Rodriguez de. 1774. Discurso sobre el fomento de la industria popular, pp. cxviii-cxix. El pasaje citado, salvo el destacado en negrita, corresponde a la cita realizada en la Aurora de Chile, 1812, tomo I nủm. 23. del 16 de julio, p. 94. Consideramos importante incluir el pasaje destacado, por la referencia ce Campomanes al fomento de las artes y oficios.

23."Estatutos de la Sociedad Económica de Amigos ceel País". En: Godoy, Pedro. 1847. Espíritu de la Prensa Chilena. Santiago, Chile: Imprenta del Comercio, p. $38 t$.

24. Jbíd., p. 392

25. bíd., p. 382

26. ibid.

27."Santiago 25 de enero en la tarde". Aurora de Chile, 1813, tomo il núm. 4. Santiago, Chile, jueves 28 de enero, p. 16.

28.Discurso iraugural que en la apertura de la Sociedad Económica de Amigos del Pais dijo su secretario D. José Antonio de irisarni". Ausora de Chile, 1813, tomo il núm. 5, Santiago, Chile, jueves 4 de febrero, p. 19

29. Legilimado institucionalmente a partił de la Constitución de la República. del año 1833.

30. Documento que fue suscrito por José María Rozas. Manuel de Salas, José Miguel de la Barra, Manuel Blanco Encalada, Bennardo de Vera y Pintado. José Ignacio Zenteno, José Gregorio Argomedo, Juan Egania, Francisco García Huidobro, Francisco Lasta, A. J. Wavell y Francisco Oíaz. Este útitimo en calidad de secretario provisional.

31. Universidad de Chite; op. cill, p. 446 .

32. bid., p. 445

33. lbíd., p. 446.

34. Fue el caso de la Escuela de Artes y oficios de Santiago, plantel tundado en 1849.

\section{Bibliogxafía}

Camponanes, Pedro Rodrigucz de. 1774. Discurso sobre el fomento de la industria popular. Madrid, España: Imprenta de Antonio de Sancha.

Campomanes, Pedro Rodrígucz de 1775. Discurso sobre la cducación popular de los artesanos y su foncnto. Madrid, Espańa: Imprenta de Antonio de Sanchit.

"Discurso inaugural que en la apertura de la Sociedad Económica de Amigos del Pais dijo su secretario D. José Antonio de Irisarri. 1813 . Aurora de Chilc, tomo Il num. 5, Santiago, Chile, jueves + de febreto.

Gandarillas. Manucl. 1812. "Concinua el Discurso sobrc la Educacion". Aurora de Chik, tomol núm. 1o, Santiago. Chile, jueves s6 de abril.

Gandarillas. Manuel. ts 12. "Educacion". Aurora de Chilc tomo l núm. 9. Santiago, Chitc. jueves g de abrit.

Godoy, Pedro. 1847. Espiritu de la Prensa Chilena. Santiago, Chile: Imprenta dd Comercio.

"Santiago 25 de Enero en la tardc". 181 . Aurora de Chilc, tomo ll nim. 4, Santiago, Chilc, jueves 28 de cnero.

Sin ritulo. 1812. Aurora de Chilc, tomo I núm. 2;, Santiago. Chilc, jucves 16 de julio.

Universidad de Chile. 1914. Escriros de don Manud de Salas y documentos rclativos a d y su familia, romo li. Santiago. Chile: Imprenea Barcelona. 